\title{
From Exoscope into the Next Generation
}

\author{
Kenichi Nishiyama, M.D., Ph.D. \\ Department of Neurosurgery, Center for Neurological Diseases, Niigata Medical Center, Niigata, Japan
}

An exoscope, high-definition video telescope operating monitor system to perform microsurgery has recently been proposed an alternative to the operating microscope. It enables surgeons to complete the operation assistance by visualizing magnified images on a display. The strong points of exoscope are the wide field of view and deep focus. It minimized the need for repositioning and refocusing during the procedure. On the other hand, limitation of magnifying object was an emphasizing weak point. The procedures are performed under $2 \mathrm{D}$ motion images with a visual perception through dynamic cue and stereoscopically viewing corresponding to the motion parallax. Nevertheless, stereopsis is required to improve hand and eye coordination for high precision works. Consequently novel 3D high-definition operating scopes with various mechanical designs have been developed according to recent high-tech innovations in a digital surgical technology. It will set the stage for the next generation in digital image based neurosurgery.

Key Words : Neuroendoscope · 3D endoscope · Exoscope · Digital image $\cdot$ Surgical technology.

\section{INTRODUCTION}

An operating microscope $(\mathrm{OM})$ has been considered as the mainstay in modern neurosurgery. It produces magnified images on the retina stereoscopically while providing detailed views of surgical anatomy. During the last two decades, neuroendoscopes are increasingly used in neurosurgery for intraventricular lesions, spine, hematoma and predominantly for trans-sphenoidal skull base surgery. An endoscope is sometimes used as an adjunct to microsurgery with OM. So-called 'endoscope-assisted microsurgery' is one of these surgical techniques ${ }^{1)}$. An endoscope can be useful by means of providing a better overview of the operating field and also revealing detailed structures with fine illumination. Surgeons visualize the operative field from a display connected to the camera with usage of an endoscope. On the other hand, compared with OM, the target is viewed only through right and left eyepieces. In 2008, an 'exoscope' system i.e. the video telescope operating monitor (VITOM) was introduced as an alternative to both OM and endoscope. The exoscope serves for observing and illuminating an object field on a patient from a position set apart from the patient's body. It enables surgeons to complete the operation under assistance by visualizing magnified images with high resolution on a display ${ }^{4,5)}$. However, VITOM provides only two-dimensional (2D) views. Therefore stereopsis was required to improve hand and eye coordination for microsurgical procedures with usage of the exoscope. Subsequently novel three-dimensional and high-definition operating scopes have been developed according to recent hightech innovations in a digital surgical technology.

- Received : February 16, 2017 • Revised : February 21, 2017 •Accepted : February 23, 2017

- Address for reprints : Kenichi Nishiyama, M.D., Ph.D.

Department of Neurosurgery, Center for Neurological Diseases, Niigata Medical Center, Kobari 3-27-11, Niigata 950-2022, Japan

Tel : +81-25-232-0111, Fax : +81-25-231-3431, E-mail : nishiken@d4.dion.ne.jp

This is an Open Access article distributed under the terms of the Creative Commons Attribution Non-Commercial License (http://creativecommons.org/licenses/by-nc/4.0) which permits unrestricted non-commercial use, distribution, and reproduction in any medium, provided the original work is properly cited. 


\section{EXOSCOPIC SURGERY WITH VITOM}

One of the strong points of VITOM is the working distance (WD). It is comparable to OM. The standard neuroendoscope can only provide a short focal distance of approximately 3 to $20 \mathrm{~mm}$. In contrast, VITOM produces 250 to $750 \mathrm{~mm}$. Focal distance is same as WD in endoscope and also in the exoscope. Therefore, VITOM allows abundant space to insert and manipulate the instruments according to this specification (Table 1).

Spinal surgery is considered as an ideal application for the exoscope $^{8)}$. Usually the VITOM is placed at regular interval of $25 \mathrm{~cm}$ from the operative field at first. Two displays were positioned face to face (Fig. 1). An illustrative case of spinal lipoma surgery with usage of VITOM was shown on Fig. 2. The main manipulations in this surgery were as follows; drilling the vertebral arches, cutting dura and arachnoid membrane with scissors, making suspending stitches, separating adherent arachnoid, resection of the lipoma along the white plane, forming circular arc by sawing the pia matter to make a neural cord and closure of dura at last. These operative techniques were completed under 2D motion images with a visual perception through dynamic cue and stereoscopically viewing corresponding to the motion parallax. But lacking the stereoscopic vision made it difficult to manipulate required handeye coordination such as taking hold the needle and stitch up. According to my clinical experiences, three-dimensional (3D)

Table 1. Specifications of the operative scopes

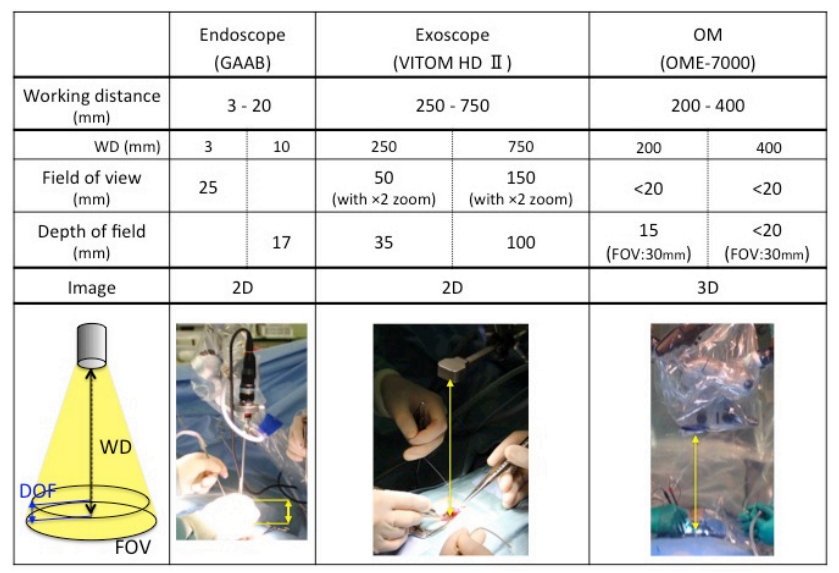

VITOM : the video telescope operating monitor, OM : operating microscope, WD : working distance, DOF : depth of field, FOV : field of view motion images must be necessary in a case with complete resection of deep seated lesions or vascular anastomosis for exoscopic surgery.

The emphasizing advantages of exoscope were regarded as the wide field of view (FOV) and deep focus. VITOM provides the ability to maintain $35 \mathrm{~mm}$ depth of field (DOF) under 50 $\mathrm{mm}$ FOV. It minimized the need for repositioning and refocusing during the procedure. On the other hand, limitation of magnifying object was the weak point. Only $50 \mathrm{~mm}$ FOV is possible under minimum WD, however, less than $20 \mathrm{~mm}$ FOV is easily available under $200 \mathrm{~mm}$ WD with OM (Table 1). From this point of view, further refinements in high resolution imaging under strong magnification, such as digital zooming would be a welcome to the VITOM.

\section{THREE DIMENSIONAL AND HIGH DEFINITION NEUROENDOSCOPE SYSTEM}

In the field of laparoscopic surgery, 3D laparoscopes with various mechanical designs are currently used successfully. Those prevent signs of fatigue while shortening the operating time and facilitate various procedures inside the body, especially in performing precise suturing. 3D endoscope provides enhancing subject depth perception and spatial orientation. According to the recent reports, 3D images improve surgical dexterity in the field of neuroendoscope ${ }^{3,10)}$. For example, clin-

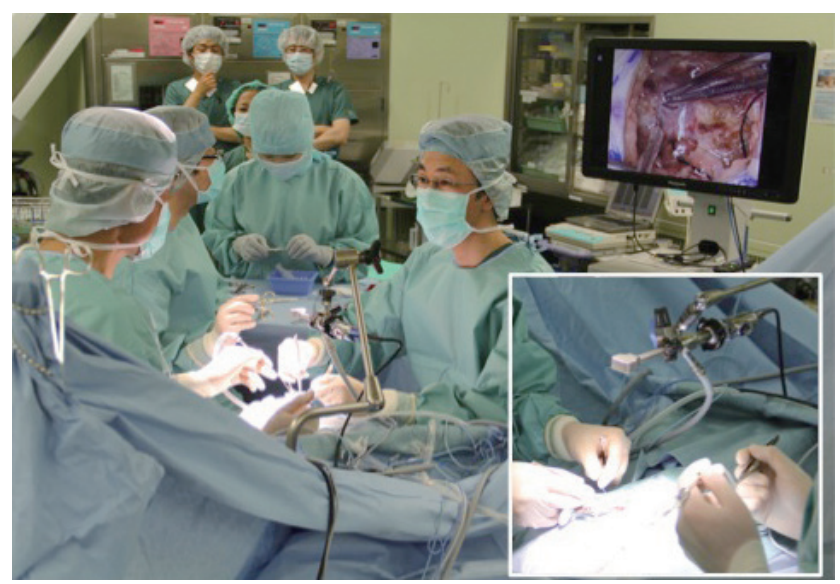

Fig. 1. Operating theater during exoscopic surgery. Setting VITOM at interval of $25 \mathrm{~cm}$ from the operative field allows abundant space for manipulation. Two surgeons operate with watching two displays placed face to face. VITOM : the video telescope operating monitor. 

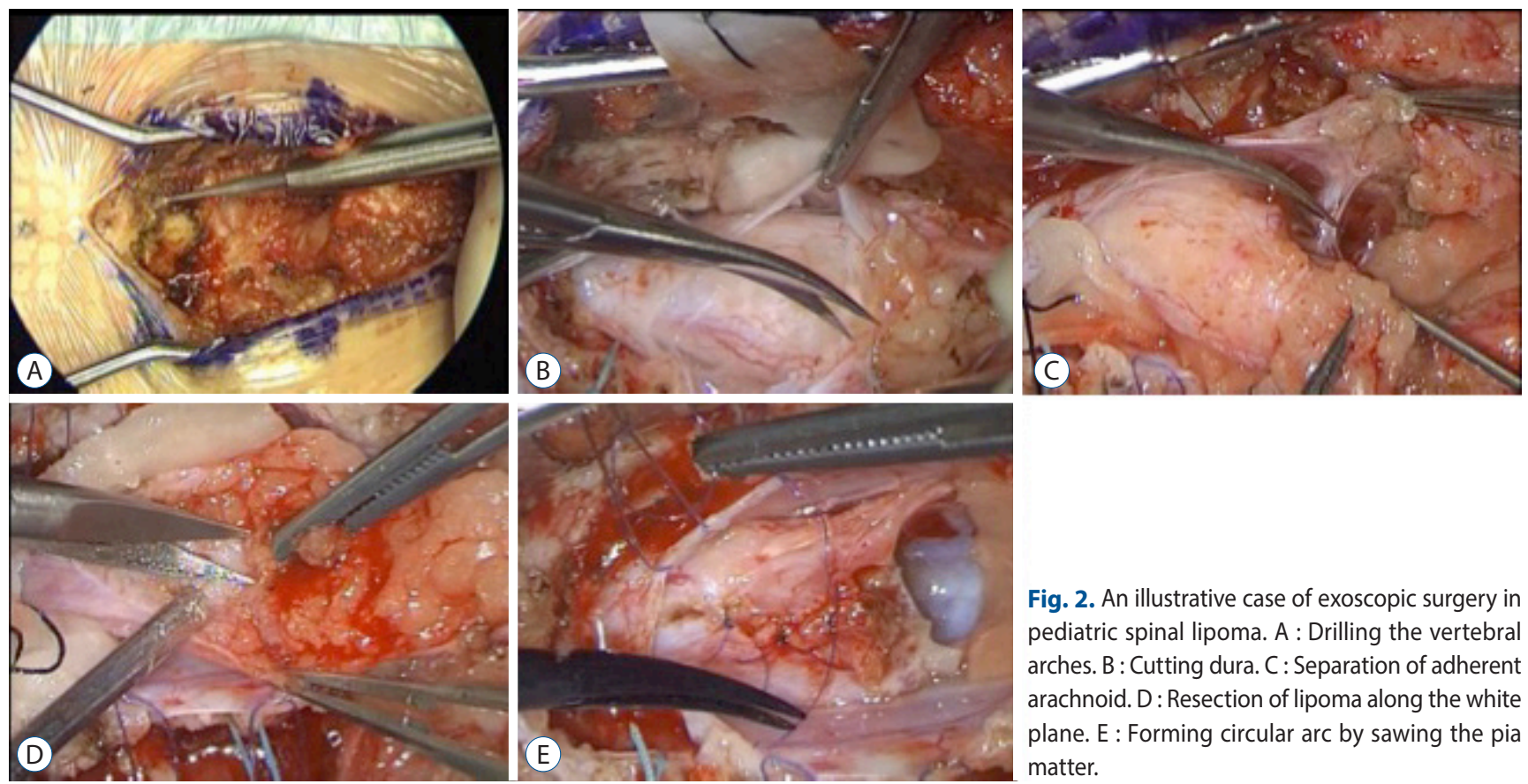

Fig. 2. An illustrative case of exoscopic surgery in pediatric spinal lipoma. A : Drilling the vertebral arches. B: Cutting dura. C : Separation of adherent arachnoid. D: Resection of lipoma along the white plane. E : Forming circular arc by sawing the pia matter.

ical application of 3D-neuroendoscope for trans-sphenoidal surgery is regarded as an effective assistive technique and supports the feasibility and safety ${ }^{9,10)}$. Surgeons sometimes observe an intracranial object field from a position set apart from the sphenoid sinus during this procedure. It seems almost like exoscopic surgery.

The commercially available 3D endoscope in neurosurgery used as 'exoscope' is quite few. VSiii system (Visionsense, Philadelphia, PA, USA) is one of the valuable one. It is based on "insect-eye technology", which involves the incorporation of a single objective lens that splits light along two paths, reconstructing a 3D image ${ }^{9}$. On the other hand, the other currently available for visualization in endoscope are based on dual optical technology. It incorporates information from two distinctive perspectives to render a single $3 \mathrm{D}$ view, similar to human vision with binocular depth perception. Hopf et al. ${ }^{2}$ reported the clinical experiences of so-called 'exoscopic microsurgery' with usage of the Einstein Vision, 3D and highdefinition (HD) laparoscope system which is designed based on dual optical technology. They concluded that the image quality was found to equal to that of the OM, but suitability for minimally invasive neurosurgical operations is inadequate. Yoshimoto et al. ${ }^{10)}$ reported their clinical experience of endonasal surgery with usage of dual optical channel 3D endoscope (developed by Shinko Optical Corporation and com- mercialized by Machida Endoscope Corporation, Tokyo, Japan) and compared stereoscopic images with VSiii system. According to that, the stereoscopic effect of dual optical channel 3D endoscope is stronger. In other words, dual optical channel 3D endoscope exhibits greater magnitude of binocular disparity, which is translated into the sense of extrusion and depth of structures.

\section{A NOVEL 3D-HD FLEXIBLE SCOPE}

In 2014, a novel 3D and HD flexible scope called 3D-EyeFlex was developed to visualize the operative field as a 3D digital image and support more accurate microsurgery ${ }^{6}$. It was introduced with an enhanced 3D-HD laparo-thoraco videoscope system (LTF- Y009, Olympus Medical Systems). The basis of mechanical design is dual optical technology. 3D-EyeFlex has two miniature-sized charge coupled device (CCD) chips at the distal end of the scope. Consequently, regardless of rigid or flexible, placing the imaging device at the tip has made "on site" processing of images without interruption possible, while maintaining consistent image quality. A $15 \mathrm{~mm}$ long rigid 3D and HD scope with a $15 \mathrm{~mm}$ outer diameter is attached to a $615 \mathrm{~mm}$-long flexible bellows. The angle of view is $80^{\circ}$, which is different compared to OM. The focus distance, 

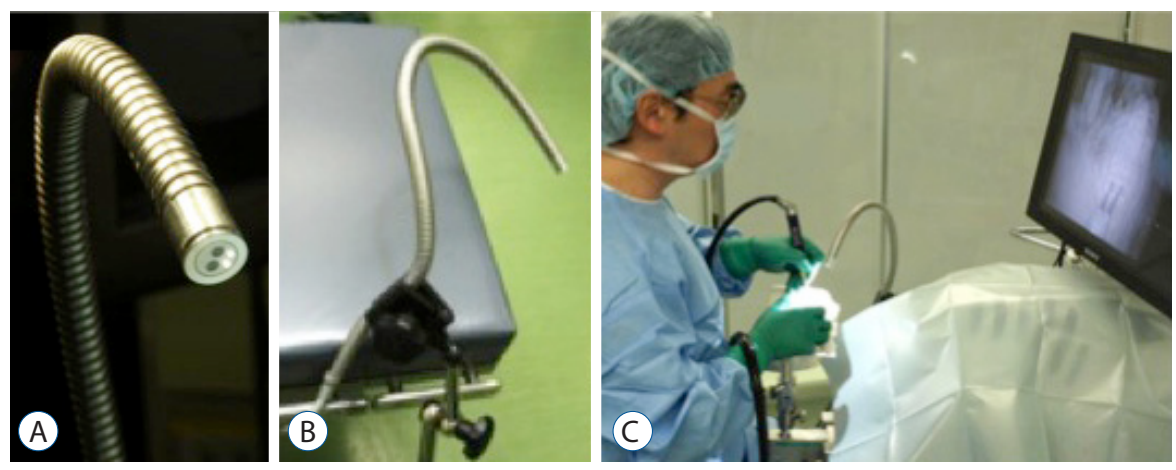

Fig. 3. 3D-Eye-Flex. A : Two mini-charge coupled devices at the distal end of the scope attached to a flexible bellows. B : 3D-Eye-Flex can be fixed to an operating table. $\mathrm{C}:$ A circular polarizing filter system is used for the 3D display to deliver life-like images. Surgeons are able to tilt their head and examine the image with wearing special lightweight glasses.

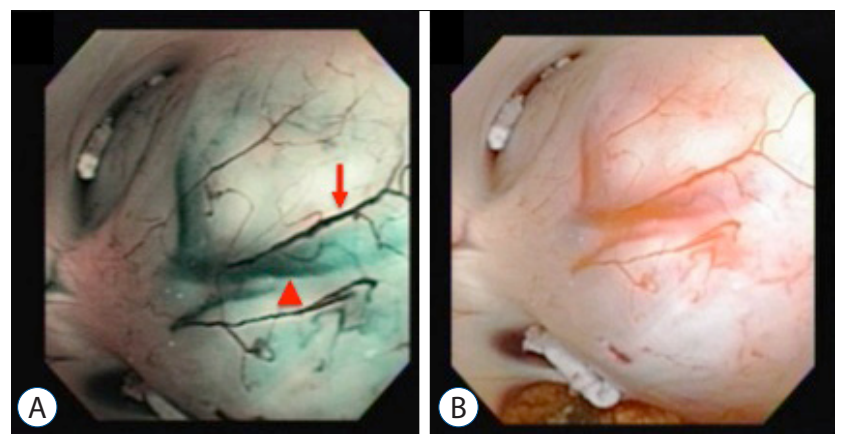

Fig. 4. A : Videoscopic findings of thalamic glioma with narrow-band imaging. An optical color separation filter narrows the bandwidth for spectral transmittance and lets only two narrow wavelengths through. These two specific wavelengths are strongly absorbed by hemoglobin. The shorter wavelengths of $415 \mathrm{~nm}$ penetrates only superficial layer, absorbed by capillary vessels in the surface and shows up brownish (arrow). While the longer, $540 \mathrm{~nm}$ light penetrates deeper, absorbed by blood vessels located subependymal layer and appears cyan on the narrow-band imaging (arrowhead). B : A standard imaging view of the same object with videoscope.

which is the same as the WD, is $18-100 \mathrm{~mm}$. The depth of field is theoretically $82 \mathrm{~mm}$ at a WD of $59 \mathrm{~mm}$. The FOV is $30-168 \mathrm{~mm}$. The illumination is not as bright as that of OM, however, the CCDs help to enhance the image. A circular polarizing filter system is used for the 3D display to deliver lifelike images (Fig. 3).

3D-Eye-Flex is considered so-called 'exoscope', an alternative to $\mathrm{OM}$, by means of capturing the 3D magnified images using digital signals from the outside of the body. The wide angle of view and large DOF inherent in the endoscope should be emphasized as advantages just like VITOM system. The large DOF of 3D-Eye-Flex drastically minimizes the need for repositioning and refocusing during a surgical procedure. Moreover, neurosurgeons feel more comfortable when using stereoscopic vision, especially in cases of deep seated lesions.
On the other hand, placing the scope close to the operative area should be required for magnifying objects. It is more likely to be stained by blood or bone dust, fogging the lens and narrowing operative space. These are the potential limitations of 3D-Eye-Flex.

\section{PROSPECT OF NEXT GENERATION IN IMAGE- BASED NEUROSURGERY}

As described above, recently developed operative scopes visualize the operative field as digital images and make it possible to verify the images according to application. For example, narrow-band imaging (NBI) while changing the light wavelength, i.e. autofluorescence, is easily applicable with 3D-EyeFlex, and can be integrated with the information obtained from white light $t^{7}$. An optical color separation filter narrows the bandwidth for spectral transmittance, lets only two narrow wavelengths through. These two specific wavelengths are strongly absorbed by hemoglobin and allow a better understanding of superficial and subependymal vasculature in the ventricle (Fig. 4). In another example, a fluorescent dye injected into the body leaks out of the tumor blood vessels due to the disrupted blood-brain barrier. It is excited with an ordinary light wavelength and the yellow color-stained tumor is visible even to the naked eye. With further refinement, an ultra-high magnified image captured by endo-cytoscopy may be possible. Currently these images of the operative field are projected onto a display adjacent to navigation images or functional images. It may be coregistered on the identical display in the near future. In state-of-the-art neurosurgery, support for various digitized information is essential for improving operative grades. Neurosurgeons could highly benefit 
from a new surgeon's eye that visualizes the operative field with integration of the medical information received by the senses and interpreted by surgeon's brain on demand. The novel operative scopes including exoscope system have potential for being developed to this new application. It will set the stage for the next generation in digital image based neurosurgery.

\section{- Acknowledgements}

The author acknowledges the support of the work regarding development of 3D-Eye-Flex by Kazunari Oka, MD and Yoshihiro Natori, MD.

\section{References}

1. Hopf NJ, Perneczky A : Endoscopic neurosurgery and endoscopeassisted microneurosurgery for the treatment of intracranial cysts. Neurosurgery 43 : 1330-1336; discussion 1336-1337, 1998

2. Hopf NJ, Kurucz P, Reisch R : Three-dimensional HD endoscopy - first experiences with the Einstein Vision system in neurosurgery. Innovative Neurosurgery 1 : 125-131, 2013

3. Inoue D, Yoshimoto K, Uemura M, Yoshida M, Ohuchida K, Kenmotsu
$H$, et al. : Three-dimensional high-definition neuroendoscopic surgery: a controlled comparative laboratory study with two-dimensional endoscopy and clinical application. J Neurol Surg A Cent Eur Neurosurg 74 : 357-365, 2013

4. Mamelak AN, Danielpour M, Black KL, Hagike M, Berci G : A highdefinition exoscope system for neurosurgery and other microsurgical disciplines: preliminary report. Surg Innov 15 : 38-46, 2008

5. Mamelak AN, Nobuto T, Berci G : Initial clinical experience with a highdefinition exoscope system for microneurosurgery. Neurosurgery 67 : 476-483, 2010

6. Nishiyama K, Natori Y, Oka K : A novel three-dimensional and highdefinition flexible scope. Acta Neurochir (Wien) 156 : 1245-1249, 2014

7. Oka K : Introduction of the videoscope in neurosurgery. Neurosurgery 62(5 Suppl 2) : ONS337-ONS340; discussion ONS341, 2008

8. Shirzadi A, Mukherjee D, Drazin DG, Paff M, Perri B, Mamelak AN, et al. : Use of the video telescope operating monitor (VITOM) as an alternative to the operating microscope in spine surgery. Spine (Phila Pa 1976) 37 : E1517-E1523, 2012

9. Tabaee A, Anand VK, Fraser JF, Brown SM, Singh A, Schwartz TH : Three-dimensional endoscopic pituitary surgery. Neurosurgery 64(5 Suppl 2) : 288-293; discussion 294-295, 2009

10. Yoshimoto K, Mukae N, Kuga D, Inoue D, Hashizume M, lihara K : Dual optical channel three-dimensional neuroendoscopy: Clinical application as an assistive technique in endoscopic endonasal surgery. Interdiscip Neurosurg $6: 45-50,2016$ 